\title{
1 Perfect representation of a convex 2D contour with only one Fourier
} component

$4 \quad$ Alf Harbitz ${ }^{*}$

$5 \quad{ }^{1}$ Institute of Marine Research, Tromsø, Norway

6*alf.harbitz@hi.no

\section{Abstract}

10 Shape analysis of a closed 2D contour is an important topic within biological shape

11 analysis, where Fourier methods to reproduce the shape with a limited number of

12 parameters have been and still are of vital importance. An example is within

13 marine management research on fish, where shape analysis of otolith (earstone)

14 contours is performed for species identification as well as for stock discrimination

15 purposes. In both cases, it is expected that the fewer parameters that are needed in a

16 method to reproduce the contour sufficiently good, the better. This contribution

17 outlines how a convex contour of any shape can be represented to any wanted

18 accuracy by only one Fourier component. The key idea is to allow a flexible choice

19 of a predetermined number of $x$-values along an $x$-axis that goes through the two

20 most distant points of the contour. The $y$-variable along the perpendicular $y$-axis is

21 then monotonically transformed to a $z$-variable so that the minium and maximum $z$ -

22 values on the contour have the same distance from the $x$-axis. The $x$-values of the

23 contour points are now chosen so that the corresponding $z$-values on the contour

24 follows a perfect sinusoid if the $x$-values were equidistant. The method is 
25 illustrated by application to lasso contours of Norwegian Coastal Cod (NCC) and

26 North East Arctic Cod (NEAC) otolith images, where the average new $x$-positions

27 for the individual otolith contours were applied to all otoliths. The results show that

28 a considerably better fit to the original individual otolith contours were obtained by

29 applying the invers FFT to the new $y$-values than by the frequently applied 2D

30 EFDs (Elliptical Fourier Descriptors) approach, for the same number, $m<11$, of

31 frequency components. A promising classification result was also obtained by the

32 linear Fisher discrimination method and cross validation applied to the individual

$33 x$-values for the NCC and NEAC otoliths, with $82 \%$ score for NCC and $80 \%$ score

34 for NEAC with sample sizes 367 and 240, respectively.

Keywords: Fourier methods, otolith contour, Elliptical Fourier Descriptors, method modification

Shape analysis of fish otolith contours for species identification, fish stock

41 discrimination and other fishery management issues has been one of many fields with a rapidly increasing activity within biological shape analysis. Though there exist several methods for such analyses, Fourier methods [1] have been and still are the major approach in terms of scientific work reported in peer-reviewed journals. Since Fourier methods handle periodic phenomena, this is a natural approach to the analysis of a closed, 2-dimensional (2D) contour as we can think of a cursor tracing the contour in e.g. an anticlockwise direction several times providing a cyclic 
record with a smooth joint between each cycle. This is contrary to $1 \mathrm{D}$ time series records that normally will have an abrupt transition if repeated.

Among Fourier methods applied to shape analysis of a closed contour, Elliptical Fourier Descriptors (EFDs) [2], has been and is the dominant approach with an exponentially increasing number of scientific papers over the last decades [3]. The method is described in more detail in the method section, but briefly speaking it consists of approximating the contour with a superposition of ellipses with different tracing frequencies. Normally 10 frequencies will be sufficient to give a very good approximation to the large-scale structure of a 2D contour, even for rather complex shapes. Strangely enough the classical EFD needs more than one frequency component to represent a pure ellipse properly. In a recent paper [4] a modified EFD method was introduced that describes a pure ellipse perfectly with only one frequency component, and in addition appears to give better large scale approximations to general contours for a given number of frequency components than do the classical EFD.

A recipe for transforming a $2 \mathrm{D}$ contour to $1 \mathrm{D}$ is given in [5], which we denote MIRR because it mirrors the lower part of the contour around a vertical axis at the maximum $x$-value of the contour. The method is described in more detail in the method section. This approach applies FFT to the transformed $y$-coordinate values and needs half as many parameters per frequency component as EFD, but ambiguities easily occur for non-convex shapes. This method also reproduces

badly a pure ellipse with only one frequency, but a modified method [4] based on a 
72 particular choice of non-equidistant $x$-values reproduces a pure ellipse virtually

73 perfect with only one frequency component. In addition, the modified method

74 appears to give better fit to the large-scale features of the original contour than the

75 original method with the same number of frequency components.

Another popular Fourier method [6] uses the tangent angle to the contour as a basic variable instead of Cartesian coordinates as used by EFDs and MIRR. The continuous contour is created by a smooth interpolation between succeeding sampled contour points and a set of contour points is created with equidistant

81 spacing along the contour. As for MIRR only two Fourier coefficients are needed

82 for each frequency component and these are independent of each other. This approach also suffers from a good reproduction of a pure ellipse based on one frequency component, though it performs much better than the original EFD and MIRR in this case.

All the mentioned Fourier methods are aimed at describing periodic phenomena, and thus are not particularly appropriate to describe enhanced features of a contour on a very local scale. In the latter case, Wavelet transform has proven to be a useful alternative to Fourier methods [3],[7]. The main objective of this paper is to introduce a Fourier method, which we denote 1FC, which can reproduce any convex shape of a contour to any degree of accuracy

94 with only one frequency component, by allowing a flexible choice of $x$-values adapted to the single contour under consideration. For non-convex shapes, the 
easily provided lasso contour coordinates [8] represent a guaranteed representation

97 of the contour in terms of a convex polygon of lasso contour coordinates. The method is applied to samples of 2D contours of whole otoliths (earstones) extracted

101 lasso contour of a whole NCC otolith is shown in Fig. 1. A direct discrimination

102 approach with 1 FC is applied by choosing a specific set of $x$-percentiles for each

107 of the individual optimal $x$-values is examined by use of the FFT of the corresponding $y$-values for each contour. The goodness of fit is calculated based on the invers FFT for different numbers of frequency components and the results are

110 compared with the classical EFD approach. It is also outlined how discrimination

111 analysis can be performed based on the FFT of the individual $y$-values

112 corresponding to the average of the characteristic average new $x$-values for each 113 stock.

115 Fig. 1. A typical NCC lasso contour with approximations by different Fourier 116 methods with 3 frequency components except in e): a) Use of the original MIRR 117 method, clearly showing false ringing features that disappear with the modified

118 MIRR in b). In d) we also see a clearly better fit by the modified EFD compared to 119 the result with the original EFD in c). In e) we see that by $1 F C$ one frequency 
120 component is sufficient to give a virtually perfect reproduction of the original

121 contour, and also a very good approximation by using the population $\mathrm{x}$-values as

122 seen in $\mathrm{f})$.

MATERIAL AND METHODS

\section{MATERIAL}

128

129 The method outlined, along with other Fourier methods to be compared with, were

130 applied to samples of NCC (367 otolith contours from the northern part of the

131 Norwegian coast) and NEAC (240 otolith contours from the south-western part of

132 the Barents Sea area). Stock discrimination results based on classical EFD methods

133 are reported for the comparisons of NCC and NEAC in [9], which also reports

134 discrimination results based on genetics for the same stocks, giving clear indication

135 of differences between the stocks. In order to avoid false discrimination because of

136 differences in fish length, the fish samples were restricted to fish lengths between

$137 \quad 30$ and $70 \mathrm{~cm}$.

138 


\section{Elliptical Fourier Descriptors (EFDs)}

143 The EFD approach consists of approximating the contour with a superposition of

144 ellipse trace vectors that trace each ellipse in a (normally) counterclockwise

145 direction with different frequencies. The original EFD method is outlined in [2]. A

146 nice feature of EFD is that there is no restriction on how the contour points are

147 chosen. Standardization of the shape is found based on the first EFD ellipse. The

148 original contour is rotated so that the major axis of the ellipse is horizontal, and

149 each EFD is divided by the length of the major ellipse axis. Further, the order of

150 the contour points are shifted so that the first point is the one closest to the

151 rightmost point on the (rotated) first EFD ellipse. Each frequency component

152 consists of 4 parameters, two characterizing the $x$-values and two characterizing the

$153 y$-values. For the standardized EFDs, 3 of the 4 EFDs for the first ellipse are

154 identical for all contours, while the fourth is the ratio between the minor and major

155 axis of the first EFD ellipse.

156

157 A disadvantage of EFD is that the four descriptors for each ellipse are dependent,

158 contrary to the two parameters describing a pure sinusoid in one dimension.

159 Another property to be aware of is that the EFD approach is very sensitive to

160 smoothing. In fact, small scale variations that normally are not visible without

161 zooming may have a dramatic effect on the low frequency (large scale) EFD

162 components. This sensitivity may easily lead to pitfalls in discrimination analysis

163 providing false discrimination results. A method to examine if such pitfalls are 
164 present is outlined in [8]. In general smoothing of the contour is strongly

165 recommended [6].

166

167 The reason why EFD performs badly on a pure ellipse is that the outline of the

168 descriptors is based on the concept of a moving cursor with constant speed. This is

169 in conflict with the classical mathematical description of an ellipse with $x=a \cdot \cos t$

170 and $y=b \cdot \sin t$ with time $t$ as parameter, with speed $v=d s / d t$ that is not constant

171 unless for a circle where $a=b$. A modified EFD method using the appropriate

172 tracing speed is given in [4] and demonstrates that a pure ellipse is now reproduced

173 virtually perfectly by only one frequency component. In addition, the modified

174 method appears to give a considerably better approximation to the large-scale

175 feature of a fish otolith contour than the original EFD with the same number of

176 frequency components up to 10 components, say.

177

178 The MIRR (partial reflection) Fourier method

180 We use the term MIRR (abbreviation for mirror) to denote the 'partial reflection'

181 concept introduced in [5]. This is a method where the 2D contour is transformed to

182 a $1 \mathrm{D}$ curve. Let $x_{\min }$ and $x_{\max }$ denote the minimum and maximum $x$-value of the

183 horizontal coordinates of the contour points. The lower part of the contour between

$184 x_{\min }$ and $x_{\max }$ is mirrored around a vertical axis through $x_{\max }$, and a set of new

185 contour points $(x, y)$ are calculated with equidistant $x$ 's between $x_{\min }$ and $x_{\max }$. Then

186 the FFT of the y-values are calculated, and a 'large scale' approximate 
187 reproduction of the 1D curve is found based on the invers FFT of the first

188 frequency components. An advantage with this approach is that the FFT components are independent of each other with only two parameters (coefficients)

190 per frequency component. To avoid ambiguities, however, the upper and lower

191 parts of the contour need to be pure functions of $x$. This is the case for convex

192 contours, which is the major focus of this paper.

193

194 For a pure ellipse with the $x$-axis along the major axis, the MIRR technique with

195 equidistant $x$ 's needs more than one frequency component to provide a good

196 approximation, because the ellipse is vertical at $x_{\min }$ and $x_{\max }$. By replacing the

197 equidistant $x$ 's with flexible ones, however, a set of $x$-values can be chosen such

198 that a pure ellipse will be virtually perfectly represented by only one frequency

199 component [4]:

200

201

$$
x_{i}=\left\{\begin{array}{lr}
x_{\text {min }}+\left(1-\cos \left(\frac{2 \pi i}{N}\right)\right) \cdot\left(x_{\text {max }}-x_{\text {min }}\right) / 2, & i=0,1, \ldots, \frac{N}{2}-1 \\
x_{\text {max }}+\left(1+\cos \left(\frac{2 \pi i}{N}\right)\right) \cdot\left(x_{\text {max }}-x_{\text {min }}\right) / 2, & i=\frac{N}{2}, \frac{N}{2}+1, \ldots, N-1
\end{array}\right.
$$

203 By the choice of $x$-values above it appears that MIRR applied to real fish otoliths

204 gives better approximations to the large scale shape than does the MIRR with

205 equidistant $x$ 's, for the same number of frequency components (ibid). 
209 The 1FC method is a generalization of the modified MIRR method described

210 above, and requires that the 2D contour can be transformed to a $1 \mathrm{D}$ curve described

211 as a function of a horizontal $x$-variable without ambiguities. This requirement is

212 satisfied for a convex contour, and in this case the transformed contour is found

213 according to the following recipe (see Fig. 2):

215 Fig. 2. The 4-step procedure to produce the transformed contour coordinates for

216 application of the 1FC method. Note that the transform in point 3) is one of a

217 whole range of actual candidates, where a linear scaling of either the positive or the

218 negative part in step 2) is the simplest approach.

1. Find the two most distant points on the contour and define the $x$-axis through these two points.

2. Rotate the contour so that the $x$-axis becomes horizontal and scale the $x$ variables so that $x_{\min }=-1$ and $x_{\max }=0$. Define the $y$-axis perpendicular to the $\mathrm{x}$-axis and let it be tangential to the transformed contour at $x_{\max }$.

3. Let $z$ denote any monotonic transform of $y$ so that $z_{\max }-z\left(x_{\max }\right)=\mathrm{z}\left(x_{\max }\right)-$ $z_{\text {min }}$, e.g. by the $\log$-transform, $z=\log (y+a)$ with $a=-y 1 y 2 /(y 1+y 2)$, as illustrated in Fig. 2, or by a linear scaling $z=y / y \max$ for $y>0$ and $z=y / y \min$ for $y<0$. 
4. If $z_{\max } \neq 1$ or $z_{\min } \neq-1$ (as in the log-transform case), transform $z$ linearly so

$$
\text { that } z_{\max }=1 \text { and } z_{\min }=-1 \text {. }
$$

232 Note that the $a$-value in the log-transform in step 3 requires that $y_{\max }>-y_{\min }$. If this

233 is not the case, just set $y=-y$ in step 3, and set $z=-z$ after step 4 .

235 When the original $(x, y)$ are transformed to new $(x, z)$ values as described above, the

236 lower part of the contour is mirrored around the $y$-axis, so that $x_{\max }=1$. The

237 concept for representation of the transformed contour to any wanted accuracy

238 based on only one Fourier component is shown in Fig. 3. The black contour is the

239 transformed original contour, and the mirrored lower part is shown by a dashed

240 black curve. The red contour is a pure sinusoid where the red contour points

$241\left(x_{\mathrm{eq}}, y_{\mathrm{sin}}\right)$ have equidistant $x$-values, $x_{\mathrm{eq}}$. We know from standard Fourier theory that

242 for equidistant $x$-values a perfect representation of a pure sinusoid is straight

243 forward as long as the period is an integer number of the distance $d x$ between two

244 succeeding $x$-values, and there are more than two points per period of the sinusoid

245 (The Nyquist criterion, see [1]). The main idea is to find the same $y_{\mathrm{sin}}$-values on the

246 transformed contour and the corresponding $x_{\text {new }}$-values. Thus the FFT will only

247 give contribution for the first Fourier component, and a perfect representation of

248 the transformed contour is obtained by combining the inverse FFT with the new

$249 x_{\text {new }}$-values. Then the new points $\left(x_{\text {new }}, y_{\text {sin }}\right)$ can be back transformed to $(x, y)$

250 coordinates on the original contour by following the 4-step recipe for coordinate

251 transformations backwards. 
253 Fig. 3. Illustration of how the new $x$-values by the $1 \mathrm{FC}$ method are found after the 254 original convex contour is transformed as illustrated in Fig. 2. The red points that 255 perform the pure sinusoid have equidistant $x$-values with a spacing $d x$ so that the 256 distance from $x_{\min }$ to $x_{\max }$ equals an integer multiplied by $d x$.

\section{The 1FC is not restricted to convex contours}

260 First of all, any non-convex closed contour can be represented by its convex

261 envelope, or lasso contour, as illustrated in [8]. In addition, the recipe for the

262 construction of $1 \mathrm{FC}$ only requires that the $y$-values after rotation of the original

263 contour (Fig. 2) has only one maximum value and one minimum value. A

264 biological example of a non-convex shape that satisfies this criterion is the tree leaf

265 shown in Fig. 4. The green contour is based on back transformation from the 1FC

266 approach with the scaled $y$-transform and based on only $m=256$ coordinate points

267 in the reproduction of the original contour with 1345 points. Note that in this case

268 we see many deviations from a convex shape.

270 Fig. 4. Illustration of a leaf with a non-convex contour, where the 1FC method can

271 be applied. First the original contour is smoothed so that no local maxima or

272 minima values of $y$ are present, just one global maximum and minimum value. The

273 upper panel shows the original contour. The mid panel shows the contour by a

274 thick black line, with the 1FC reproduced contour in green with $256 \mathrm{x}$-values. The 
275 lower panel shows the original image with the green patched area defined by the

276 1FC coordinates.

The 1FC applied to a population of contours

280 A convex contour can be represented to any wanted accuracy with only one Fourier 281 component by the $1 \mathrm{FC}$ with a sufficiently large number $n$ of $x_{\text {new }}$ values. How large $282 n$ that is needed will depend on the shape of the contour as well as the location of 283 the original $(x, y)$ points on the contour. Some extra variables are also needed for 284 the back transform process. So to assess if the 1FC method is useful as a data285 reduction technique for individual contours as compared to other techniques like 286 the use of a chain code [2], EFD or other, has to be done by experiments.

288 Say, however, that a perfect reproduction is not needed, but rather a decent 289 reproduction that is sufficient to give the main characteristics for a population 290 under study. A population in this context could for example be the sample of all 291 otolith contours for a specific species. For the 1FC we imagine that a random 292 sample of $n$ individual contours are analyzed providing $n$ distinct samples of 293 standardized $x_{\text {new }}$-values. Assume we have decided to construct $m x_{\text {new }}$-values for 294 each contour, $m=2048$ being a typical example. We let the average of these $m$ 295 values, taken over all inidividual contours, be an estimated representation for the 296 population under study. 
298 Once a set of characteristic $x_{\text {new }}$-values are created, we can examine how efficiently

299 the individual contours can be represented based on this. First the $y$-values on the

300 contour that correspond to the new $x$-values are calculated, then an FFT is applied

301 to these new $y$-values. Thereafter, an inverse FFT is applied to the FFT with only a

302 given number, $k$, of components, and the rest set to zero. Finally, the inverse FFT is

303 back transformed to the original contour coordinates, and we measure how well the

304 original contour is being represented as a function of the number of frequency

305 components involved. Such results can be provided for different transforms within

306 the 1FC method (e.g. the log- and linear transforms) as well as with other Fourier

307 methods, to find the best method. Here we limit the attention to the comparison

308 between the linear and log transform within 1FC, and comparison with EFD.

The application of 1FC in discriminant and classification analysis

312 Say that we want to apply the shape of the outer contour of a fish species, or a fish

313 stock to find a measure to separate two populations, e.g. contours belonging to two

314 different species of fish, or two different stocks of one species. We will consider

315 two approaches, where the first and simplest one is to use a set of ranked x-values

316 among the new ones provided by the 1FC method, as descriptors. As an example,

317 let $x_{\text {new }(1)}<x_{\text {new }(2)}<\ldots<x_{\text {new (n) }}$ denote the new $x$-values in increasing order. As a

318 typical example let $n=2048$, and apply each $20^{\text {th }}$ value: $x_{\text {new(24) }}, x_{\text {new(64) }}, x_{\text {new }(84)}, \ldots$,

$319 x_{\text {new(2024) }}$ as the descriptors for each otolith. If we now have $n_{1}$ descriptors from one

320 group with known identity, and $n_{2}$ from another, we can for example apply Fisher's

321 linear discrimination method [10] directly and use cross validation (leave one out at 
322 a time) to examine how well these $x$-descriptors classify each otolith. We have no

323 general recipe for how to choose the optimum number of $x$-predictors, and their

324 corresponding order/percentiles, so this must be done by trial and failure. Some

325 ideas might be created by comparing the average $x$-values for the two groups.

327 The other discrimination approach is to find the average of the two averaged $x$ -

328 values for each group, apply them to each otolith and find the corresponding $y$ -

329 values on the contour, and calculate the FFT of these new $y$-values. Fisher's linear

330 discrimination method can then be applied on a limited number of frequency

331 components from the FFT and give an indication of discrimination success.

336 The data to be used are the original contour coordinates $\left(x_{\mathrm{org}}, y_{\mathrm{org}}\right)$ and the set of $\mathrm{m}$

337 points $\left(x_{\mathrm{fit}}, y_{\mathrm{fit}}\right)$ on the approximate contour. As a measure of accuracy we calculate 338 the mean distance between each $\left(x_{\text {fit }}, y_{\text {fit }}\right)$ point and the closest point, $\left(x_{\text {orgi }}, y_{\text {org }}\right)$, on

339 the original contour, i.e. the set of continuous lines between all succeeding original 340 coordinate points:

$$
D_{f i t}=\frac{1}{m \sqrt{A}} \sum_{j=1}^{n} \min _{\left(x_{\text {org }}, y_{\text {org }}\right)}\left(\sqrt{\left(x_{f i t j}-x_{\text {org }}\right)^{2}+\left(y_{f i t j}-y_{\text {org }}\right)^{2}}\right)
$$


344 where $A$ is the area enclosed by the closed contour and its role in the equation

345 above is to make the fitness measure invariant to unit (or scale). The role of

346 contour points and fitness points can easily be changed, and should give very

347 similar results. If not, this indicates that the resolution is too coarse. As a measure

348 of method goodness we apply the average $D_{\text {fit }}$ value over all contours belonging to

349 the group studied, e.g. the NCC sample.

353 Closely related to the classification of a group identity for an individual contour is

354 the estimator for the proportion of the numbers belonging to the group relative to

355 all other similar groups of interest. In the cod example studied it is particularly

356 important to estimate the number of NCC, because this is considered to be a

357 vulnerable stock. To do this one has to separate NCC from NEAC, which is done

358 subjectively by visual otolith inspection. In principle this could be done

359 automatically by shape analysis of otolith images. The direct estimate based on the

360 detected fraction of NCC will be biased, however, unless the probability of correct

361 classification is $100 \%$ for either stock. If we know the correct classification rates, a

362 close to unbiased estimator for the unknown proportion $p$ is as follows [11]:

363

$$
\hat{p}=\frac{N_{C C, \text { obs }} / N-\left(1-p_{N C}\right)}{p_{C C}-\left(1-p_{N C}\right)},
$$


366 where $N_{C C, \text { obs }}$ is the number of classified NCC, and N is the total number of NCC

367 and NEAC. Further, $p_{N C}$ is the probability of correct classification of a NEAC

368 individual and $p_{C C}$ is the probability of correct classification of a CC individual.

369 This estimator is further discussed in the Conclusion and Discussion section.

371 RESULTS

\section{GOODNESS OF FIT}

374 The described 1FC method was applied to the sample of $n_{1}=367$ NCC otolith

375 lasso contours and $n_{2}=240$ NEAC otolith lasso contours from images of the whole

376 otolith, where $m=2048$ standardized $x$-values were calculated for each otolith

377 giving a virtually perfect reproduction of each otolith based on only one Fourier

378 component. Then the average $x$-values for each of the two groups were applied and

379 the corresponding $y$-values on each otolith contour in the group were calculated.

380 Fig. 5 shows the goodness of fit results by applying the averaged $x$-values and the

381 approximate $y$-values based on the invers FFT of the new $y$-values on each otolith,

382 as a function of the number of frequency components. Further, the results are

383 calculated for both the log-tranform and the linear transform within the 1FC

384 method, as well as by the classical EFD method.

386 Fig. 5. Results of 1FC reproduction of 367 NCC and 240 NEAC contours based on

387 the average $x_{\text {new }}$ values for each stock, compared to the EFD results (upper two 388 curves). We see that the 1FC provides better results than EFD for the number of 
389 frequency components up to 10 . The differences between the different $1 \mathrm{FC}$ results

390 are hardly visible (log and linear $y$-transforms).

392 As we see from Fig. 5, the 1FC method performs much better than EFD with few

393 parameters, and remain better until $n_{\text {freq }}=10$. For larger $n_{\text {freq }}$ values it is hard to see

394 any difference between the methods. Though there are small differences between

395 the 1FC approaches, the linear transformation of $y$-values give better results than

396 the log-transformation, and the goodness of fit values are best for NCC for the $n_{\text {freq }}$

397 values (2-5) where the difference between NCC and NEAC is largest.

401 By some trial and failure, the best classification of NCC and NEAC by Fisher's

402 linear discrimination and cross validation, based on the new $x$-values $(\mathrm{m}=2048)$,

403 was found for the 52 ordered values $x_{\text {new(2:2:10) }}, x_{\text {new (74:50:2024) }}, x_{\text {new(2037:2:2047) }}$ with a

404 probability of $82.83 \%$ correct score for NCC and $80.83 \%$ correct score for NEAC.

406 When the average of the average new $x$-values for NCC and NEAC were applied,

407 the scores were $81.74 \%$ and $77.92 \%$ for NCC and NEAC, respectively, based on

408 the 9 first frequency components. When adding the ratio between the max and min

$409 y$-value with the $\mathrm{x}$-axis through the most distant contour points, along with the ratio

410 between the maximum extension in the $y$-and the $\mathrm{x}$-direction, as descriptors, the

411 scores increased to $85.56 \%$ and $82.50 \%$ for NCC and NEAC, respectively. These 
412 figures are not far from the scores $88.56 \%$ and $82.92 \%$ obtained by the classic

413 EFDs based on 10 frequency components. The results based on 1000 bootstrap

414 simulations are shown in Fig. 6. The average scores based on the data from which

415 the bootstrap samples are drawn are shown as white vertical lines, and indicate

416 some bias in some of the cases.

418 Fig. 6. Discrimination results with $1 \mathrm{FC}$ and EFD applied to $367 \mathrm{NCC}$ and 240

419 NEAC otolith contours based on 1000 bootstrap simulations. The $y$-values where

$420 \operatorname{FFT}(y)$ is applied are the $y$-values on the transformed contour for the $x$-values being

421 the average of the mean NCC and NEAC new $x$-values. The $y / x$ ratios are the two

422 ratio measures based on $y_{\max } / y_{\min }$ as well as the $\left(y_{\max }-y_{\min }\right) /\left(x_{\max }-x_{\min }\right)$ values. The

423 white vertical bars show the scores from data, which indicate biased values in some 424 cases.

426 CONCLUSION AND DISCUSSION

428 An easily applicable Fourier method, 1FC, is outlined that reproduces a contour

429 described by a convex polygon of coordinate points to any wanted accuracy based

430 on only one frequency component. The basic idea is to allow a flexible choice of

431 coordinate $x$-values optimally adapted to the individual contour under

432 consideration. To compare the performance of this method to reproduce the

433 contour with other methods, like EFD, the average of the optimal $x$-values adapted

434 to each contour in a particular group to be studied is applied to each contour in the 
435 group. Then an FFT is applied to the corresponding contour $y$-values, and an

436 approximate reproduction of the contour is provided by back-transformation of the

437 inverse FFT of the transformed $y$-values of the contour points. The results applied

438 to the lasso contours of whole NCC and NEAC otoliths give far better

439 approximations to the original lasso contours by 1FC than by EFD for the same

440 number of frequency components until about 10, say, then the results merge.

442 Despite the much better fit to the large scale features of a contour that was obtained

443 by $1 F C$, the discrimination results did not appear to be better than by EFD, though

444 they were optimistically high. Because of the standardization of both the $x$ - and $y$ -

445 coordinates by the $1 \mathrm{FC}$ method, possible shape differences characterized by

446 different ratios between $y$ - and $x$-values after rotation, but before scaling, are not

447 taken appropriately into account. This was indicated by the substantial

448 improvement based on the FFT of the transformed y-values when the two ratio-

449 measures for the rotated (but not scaled) contour were added to the Fourier

450 descriptors.

452 One would expect that a combination of methods with descriptors that detect

453 different shape differences between two groups would have the potential to

454 improve discrimination compared to the single method giving the best

455 discrimination. Unfortunately such results were not obtained by combining the

456 EFD descriptors and the 1FC descriptors applied to NCC and NEAC. An

457 explanation to this can be that the two methods are not different enough with 
458 regard to the actual shape studied, and that the success of combining methods may

459 vary greatly with shape.

461 It is a promising result that the set of $x$-values providing the "perfect" reproduction

462 of a contour with only one frequency component could be used directly as a pool of

463 descriptors that gave good discrimination results in our case. The results, however,

464 were sensitive to the number of $x$-values that was chosen along with their order

465 (percentile values). This might be an interesting field for more thorough studies.

467 Another field for further exploration is to study the estimator of the proportion of a

468 group. Within fisheries management of for example Norwegian Coastal Cod,

469 which is a vulnerable stock, it is important to detect the fraction of NCC in catches

470 with cod. This is done by subjective visual inspection of the otoliths today, but can

471 in principle be done by automatic image analysis of the whole otolith. If the correct

472 classification rate is known for both NCC and NEAC, the ratio based on detected

473 stocks will be biased and can be replaced by the close to unbiased estimator given

474 by Eq. 3. If we have different methods, like EFD and 1FC, giving different, but

475 close to unbiased results, these can be combined and hopefully reduce the variance

476 of the estimator.

478 The 1FC method is based on an $x$-axis going through the most distant points of the

479 original contour. In cases that this is not optimal one can play with a transformation

480 of the coordinates so that the most distant points in the transformed space appear to 
bioRxiv preprint doi: https://doi.org/10.1101/2020.09.24.311316; this version posted September $24,2020$. The copyright holder for this preprint (which was not certified by peer review) is the author/funder, who has granted bioRxiv a license to display the preprint in perpetuity. It is made available under aCC-BY 4.0 International license.

481 be more appropriate. A simple example of this is a scaling of one of the two

482 coordinates. 


\section{REFERENCES}

486 [1] Stearns SD. Digital Signal Analysis. Hayden Book Company, INC.

487 Rochelle Park, New Jersey; 1975.

488 [2] Kuhl FP, Giardina CR. Elliptic Fourier Features of a Closed Contour.

$489 \quad$ Computer Graphics and Image Processing. 1982; 18: 236-258.

490 [3] Lestrel PE. From Elliptical Fourier Functions to Wavelets: Three Decades of Boundary Morphometrics. Proc. $4^{\text {th }}$ Int Symp Biol Shape Anal (ISBSA).

493 [4] Harbitz A. (2016) Parameter-sparse modification of Fourier methods to analyse the shape of closed contours with application to otolith outlines. Marine and Freshwater Research. 2016. Available from: http://dx.doi.org/10.1071/MF15087 .

497 [5] Reig-Bolãno R, Marti-Puig P, Lombarte A, Soria JA, Parisi-Baradad V. A new otolith contour descriptor based on partial reflection. Environ Biol Fish. 2010; 89: 579-590.

500 [6] Haines AJ, Crampton JS. Improvements to the Method of Fourier Shape Analysis as applied in Morphometric Studies. Palaeontology. 2000; 43(4): 765-783.

503 [7] Sadighzadeh Z, Valinassab T, Vosugi G, Motallebi AA, Fatemi MR, $504 \quad$ Lombarte A, Tuset VM. Use of otolith shape for stock identification of John's snapper, Lutjanus johnii (Pisces: Lutjanidae), from the Persian Gulf and the Oman Sea. Fisheries Research. 2014; 155: 59-63. 
508 [8] Harbitz A, Albert OT. Pitfalls in stock discrimination by shape analysis of

509 otolith contours. ICES Journal of Marie Science. 2015; 72(7): 2090-2097.

510 [9] Stransky C, Baumann H, Fevolden SE, Harbitz A, Høie H, Nedreaas KH,

511 Salberg, Skarstein TH. Separation of Norwegian coastal cod and Northeast

512 Arctic cod by outer otolith shape analysis. Fisheries Research. 2008; 90:

$513 \quad 26-35$

514 [10] Johnson RA, Wichern DW. Applied Multivariate Statistical Analysis. $5^{\text {th }}$ ed.

$515 \quad$ Prentice Hall, Upper Saddle River, New Jersey 07458; 2002.

516 [11] Berg E, Sarvas TH, Harbitz A, Fevolden SE, Salberg, AB. Accuracy and

517 precision in stock separation of north-east Arctic and Norwegian coastal

518 cod by otoliths - comparing readings, image analyses and a genetic

519 method. Marine and Freshwater Research. 2005; 56. 
a) Original MIRR $n_{\text {freq }}=3$ c) Original EFD $n_{\text {freq }}=3$ e) 1FC, individual $x$-values $n_{\text {freq }}=1$ b) Modified MIRR

$$
n_{\text {freq }}=3
$$

d) Modified EFD

$$
n_{\text {freq }}=3
$$

f) 1FC, population $x$-values

$$
n_{\text {freq }}=3
$$


1) Find $x$-axis through 2 most distant points
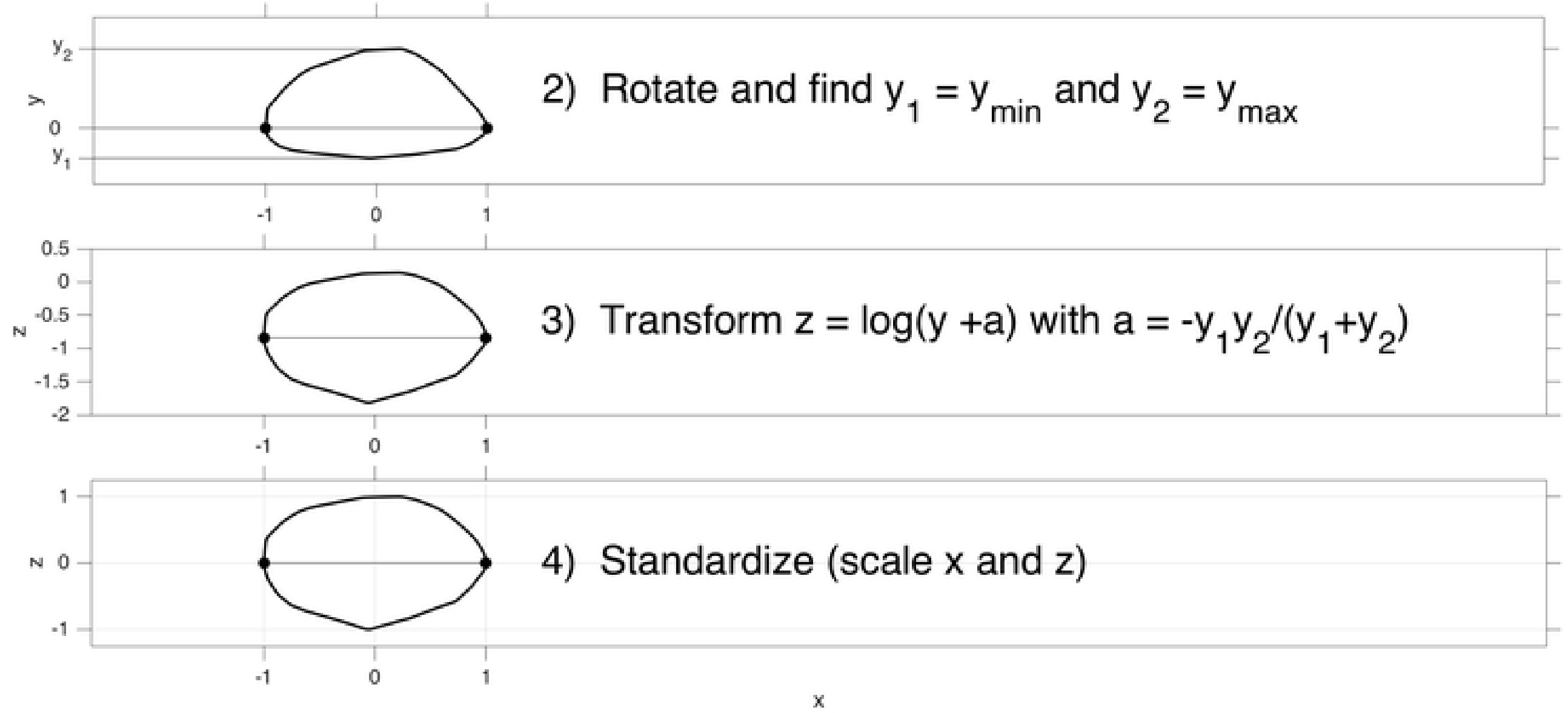

Figure 


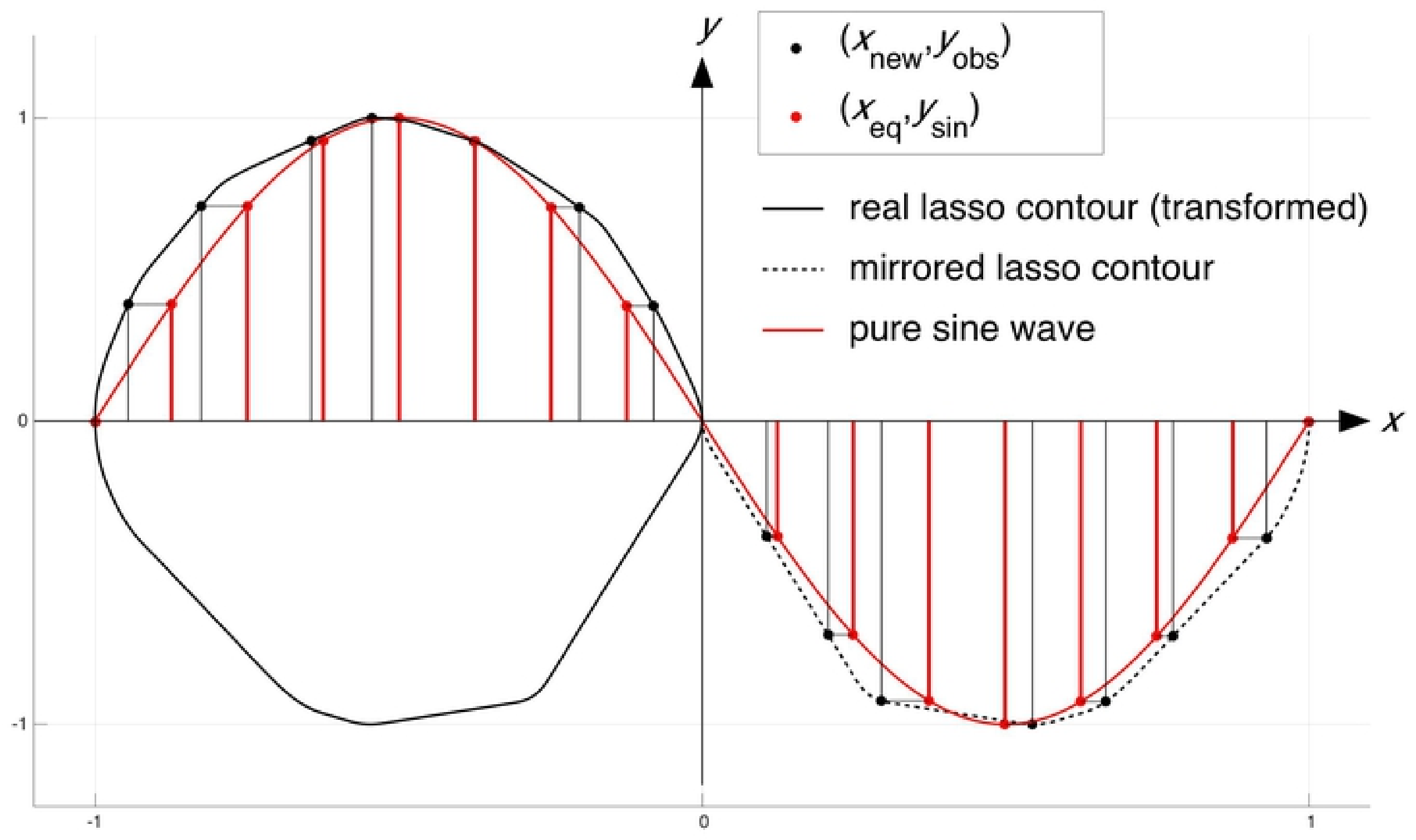

Figure 

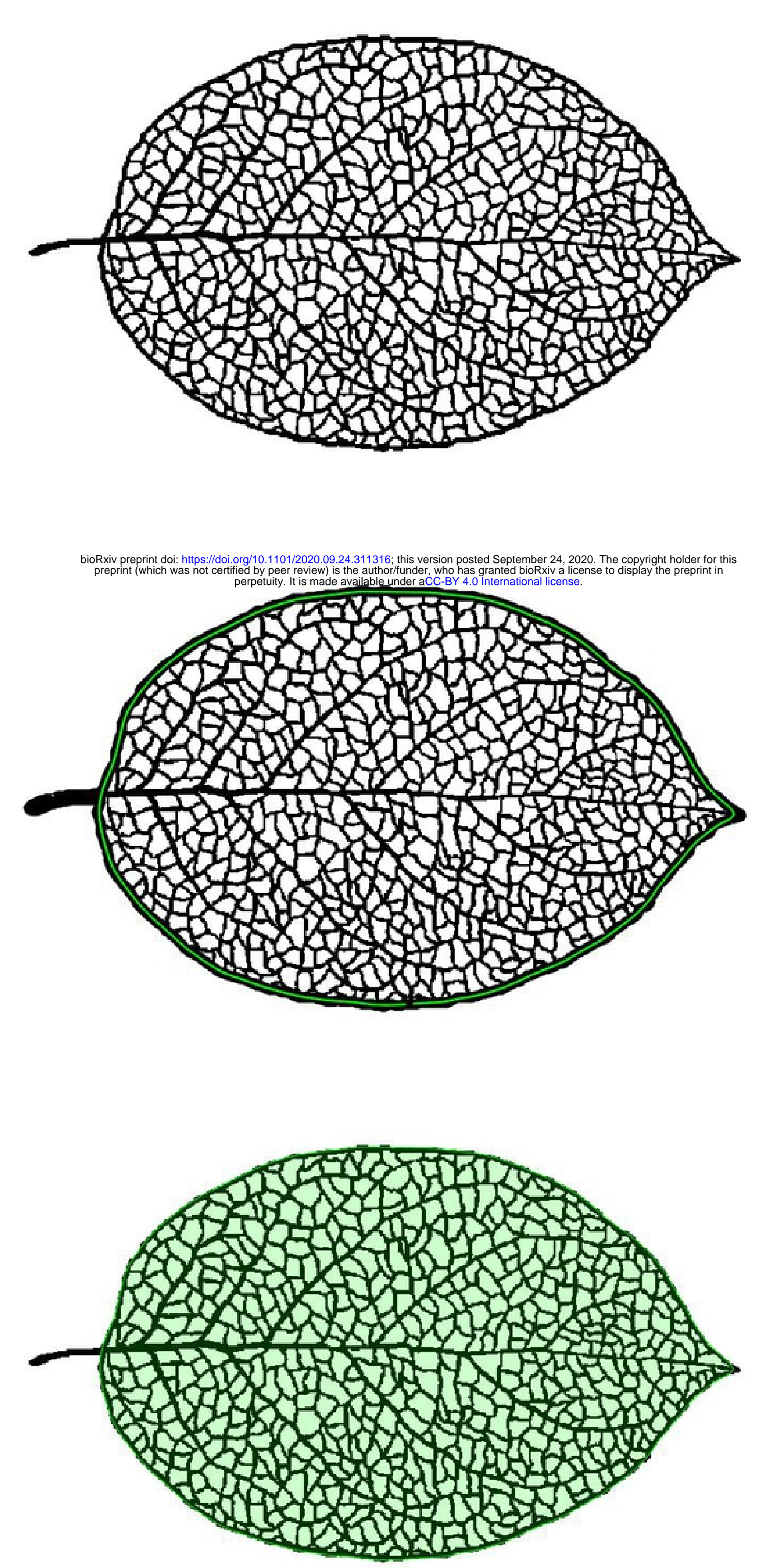

Figure 


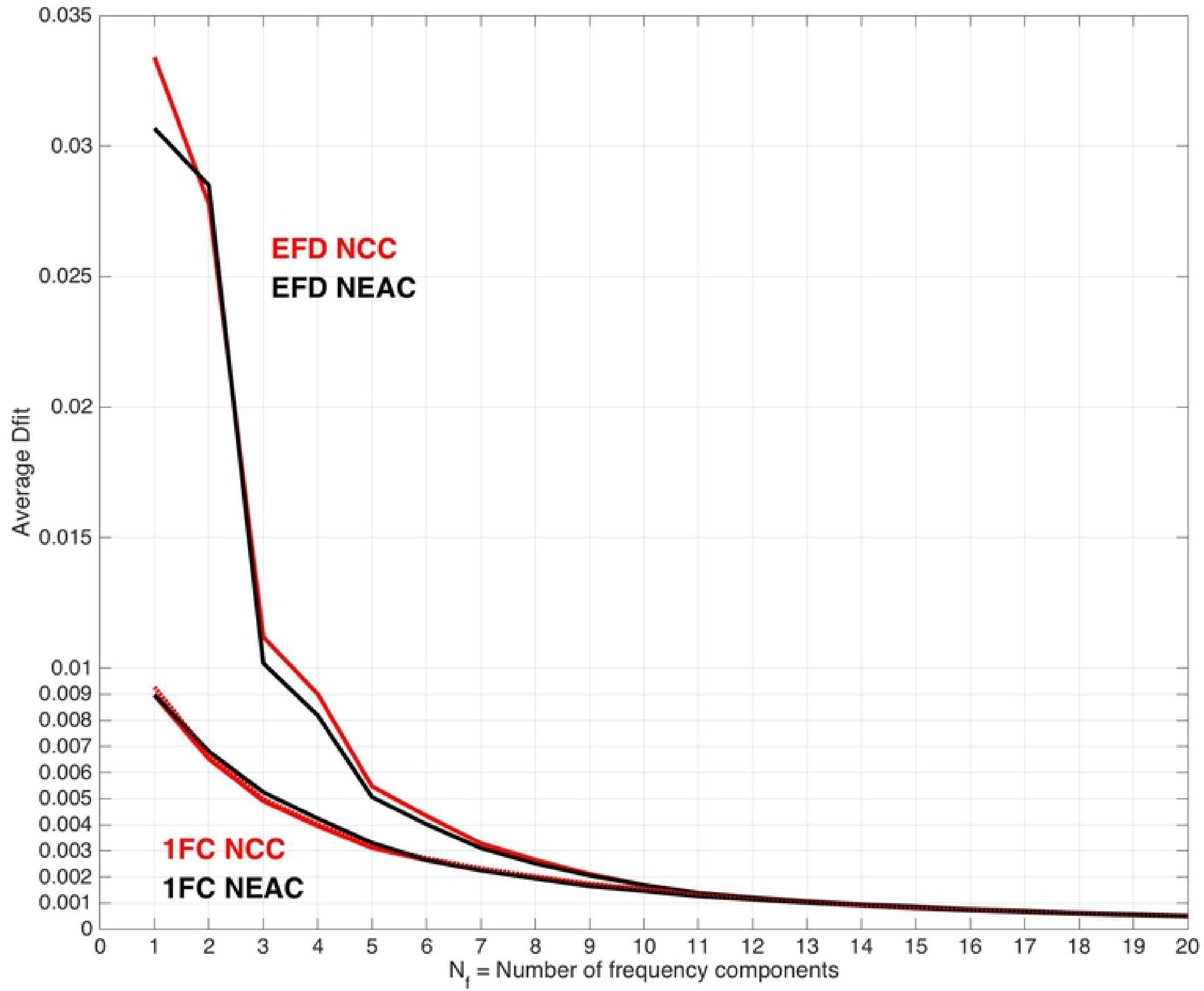

Figure 


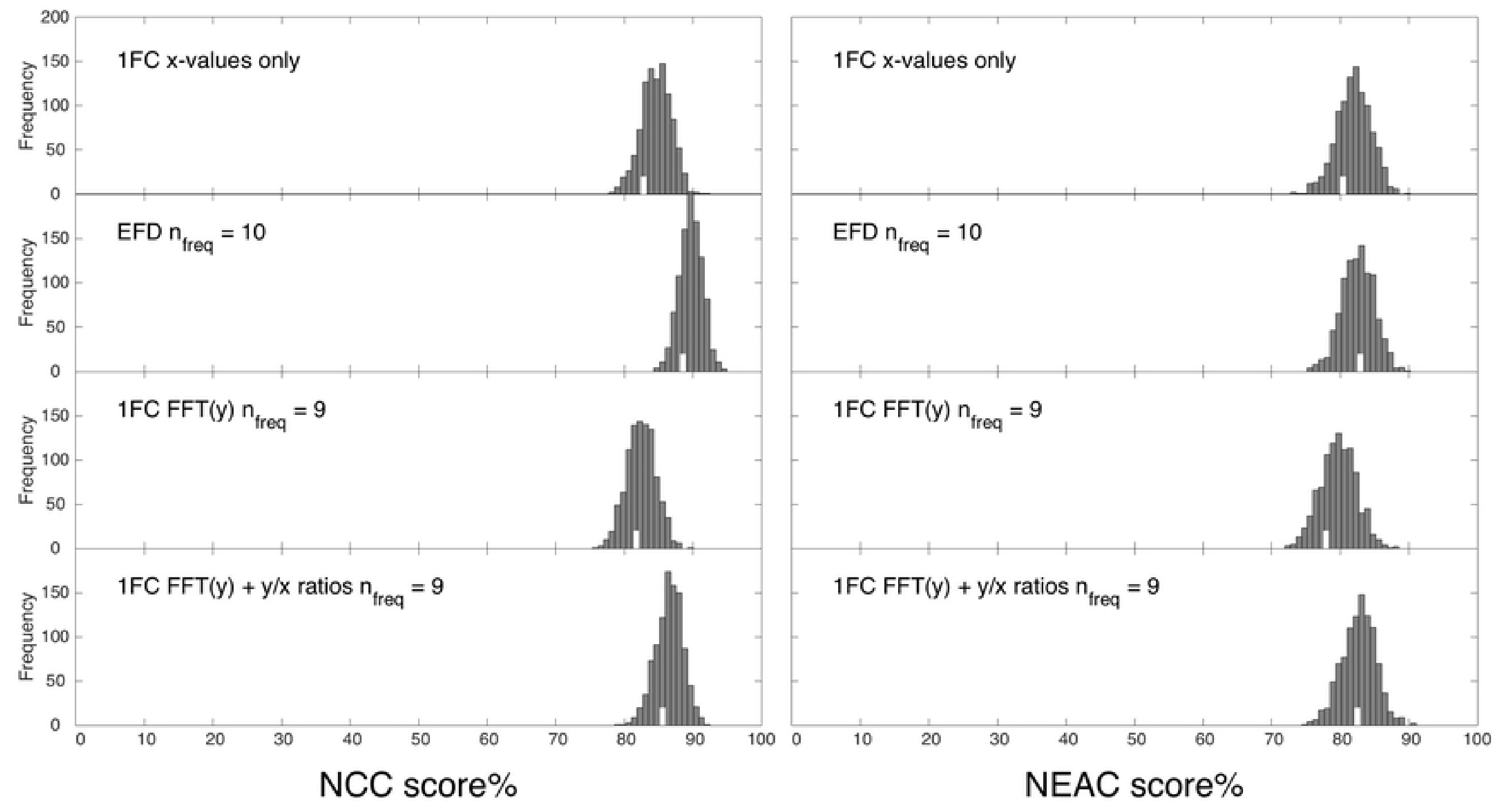

Figure 